\section{STANDARDS OF CARE}

Professionals are sometimes unaware of patients' perceptions of their practices. A survey was conducted in five short stay hospitals in France. Twenty standards of care dealing with patient information were assessed by patients and health professionals, who rated statements regarding patient care by expressing agreement or disagreement. Overall, patients' and professionals' views on what was best or worst were similar, but some differences in the perception of care were also observed. For example, the common wisdom among many practitioners is that pain management is inadequate and requires more concern. However, patients were far more satisfied with this care than the professionals. Everyone agreed that consent was requested before surgery and that visitors were asked to leave the room before an examination, but patients felt that more attention should be paid to non-technical aspects of care, to information after discharge, and to help with psychosocial problems if high quality health care is to be achieved. See p198

\section{PROBABILISTIC RISK ASSESSMENT}

Health care carries risk to patients. Some patients are harmed and a few die as a consequence of the care they receive. An important step towards making care safer is understanding how health care works as a "system". One approach is to use probabilistic risk assessment (PRA), a technique that has been used to assess the designs of high hazard, low risk systems such as nuclear power plants. PRA examines events that contribute to adverse outcomes through the use of event tree analysis and determines their likelihood through fault tree analysis. PRA complements other methods that are already in use within the patient safety community by accounting for the more complex causal interrelationships that are typical in healthcare settings and enabling the analyst to examine the effectiveness of potential solutions through direct graphical representations. However, PRA can simplify real world complexity which often lacks adequate probability data, and relies on expert assessment for deep domain knowledge. Such knowledge necessarily comes from research into the deep structure of work that is performed at the "sharp end" of acute care.

See p206

\section{FINANCIAL INCENTIVES TO IMPROVE CARE QUALITY}

The UK government is introducing a radical scheme of incentives to encourage specific improvements in care, as part of a new payment system for general practitioners. Substantial health gains could result if this approach stimulates an increase in the use of effective interventions for the cardiovascular conditions targeted by the incentives. The main impact is likely to be achieved through the control of high cholesterol levels and high blood pressure. However, the impact of achieving targets for other cardiovascular interventions will only be small because the majority of these patients are already being treated with recommended medications.

See p191

\section{CLINICIANS, LIBRARIANS, AND PATIENT SAFETY}

Information plays a significant role in both the delivery of safe medical care and within the larger culture of an organisation. Identifying professionals in health care who have the knowledge and skill to enhance information initiatives is imperative to crafting creative solutions that yield a higher level of reliability. The role of librarians as knowledge managers in healthcare organisations is highlighted. Encouraging healthcare executives and clinicians to seek partnerships with this group of professionals to enhance information delivery on safety related issues, the author provides suggestions for both institutions and the healthcare community to foster improvements.

See p218

\section{EDITOR'S CHOICE}

Effective communication between patient and practitioner is vital. Patients need to be able to let doctors and nurses know how they feel and to be able to articulate concerns and queries. Clinicians, on the other hand, need to find ways of informing patients about available choices and, when a treatment has been decided, to make sure that the patient fully understands not only the risks and hazards but also how to take the medicine. Two papers (see $p 12$ and $p 38$ ) in this issue provide further evidence of the communication gap that often exists between patient and practitioner. Perhaps it is not surprising that patients tend to differ in opinion from healthcare professionals about what is important; caregivers are more likely than patients to give priority to the "technical" aspects of care. Maybe that is as it should be. But it seems that when initiating medication for chronic conditions, practitioners are not that good at giving patients crucial information, with the consequence that more patients than is often realised do not take medicines as directed. This surely must count as a technical aspect of care? If healthcare professionals made it a priority to properly understand their patients' concerns, inform them effectively about the technical aspects of care, and provide timely opportunity for asking questions, perhaps more patients would have the confidence either to take prescribed medication, or at least tell someone when they had decided not to do so. It all seems so simple. But another paper (see $\boldsymbol{p} 9$ and $p 16$ ) has demonstrated that we know little about how best to inform patients about the risks associated with medicines. Patient centredness has become one of those tired phrases that has ceased to have much impact or meaning; maybe this is because we do not yet have the knowledge and the tools to put this ideal into practice. 